\title{
A Proximity and Pressure Touch Screen Using Separation-Type Electrodes
}

\author{
Satoshi Tsuji ${ }^{a,}$, , Teruhiko Kohama ${ }^{\mathrm{a}}$ \\ aFukuoka University, 8-19-1 Nanakuma, Jonan-ku, Fukuoka 814-0180, Japan, \\ *Corresponding Author: tsuji@fukuoka-u.ac.jp
}

\begin{abstract}
Touch screens are widely used in recent year. Most touch screens detect contact position only. We propose the proximity and pressure touch screen. This touch screen consists of plane separation-type electrodes and parallel-type electrodes with mutual capacitance measurement. The proposed sensor detects object using plane separation-type electrodes at proximity range. In addition, the sensor detects a pressure and precise position using plane separation-type electrodes and capacitance pressure sensor which is paralleltype electrodes on the contact. We think that the proposed proximity and pressure touch screen will be a functional and useful interface.
\end{abstract}

Keywords: Touch screen, Proximity and pressure sensor, Mutual capacitance measurement.

\section{Introduction}

In recent year, touch screens are widely used for interfaces between humans and device. Various sensing techniques have been developed for touch screens ${ }^{(1-4)}$. Among these approaches, mutual capacitance measurement has been used mainly for small touch screens such as smartphones and tablet devices because it can detect multitouch events ${ }^{(5)}$. Most previous touch screen detect the contact position between the touch screen and the object. On the other hand, proximity touch screens have been proposed to detect finger before contact. Using a camera to detect the finger has also been proposed ${ }^{(6)}$. However, this device become large because an external camera is required. Thus, it is difficult to use for small mobile devices. For small mobile devices, the method of attaching an infrared sensor to the side of a smartphone has been proposed to detect the movement of a hand ${ }^{(7)}$. However, it is difficult to accurately detect the position of a finger on the display. A method combining self-capacitance measurement and mutual capacitance measurement to detect fingers within the proximity range and their contact position has been presented ${ }^{(8)}$. However, it is difficult to detect multi-point touch using self-capacitance measurement because 'ghosts' occur ${ }^{(5)}$, and it is not considered to detect pressure.

On the other hand, the pressure touch screens have been proposed to detect the pressure. Using some pressure sensor attaching the smartphone to detect the pressure condition is proposed ${ }^{(9)}$. In addition, the smartphone ${ }^{(10-11)}$ which can detect the contact position and pressure by pressure sensor and touch screen were on sale. However, it is not considered to detect the object at proximity range.

The goal of our research is to develop a proximity and pressure touch screen using mutual capacitance measurement that detect an object within the proximity range, contact position, and pressure for operation of the display of the device. Fig. 1 shows the goal image of a proximity and pressure touch screen on a display. When a finger approaches the touch screen, the displayed image is enlarged according to the distance between the finger and the sensor. Thus, it is

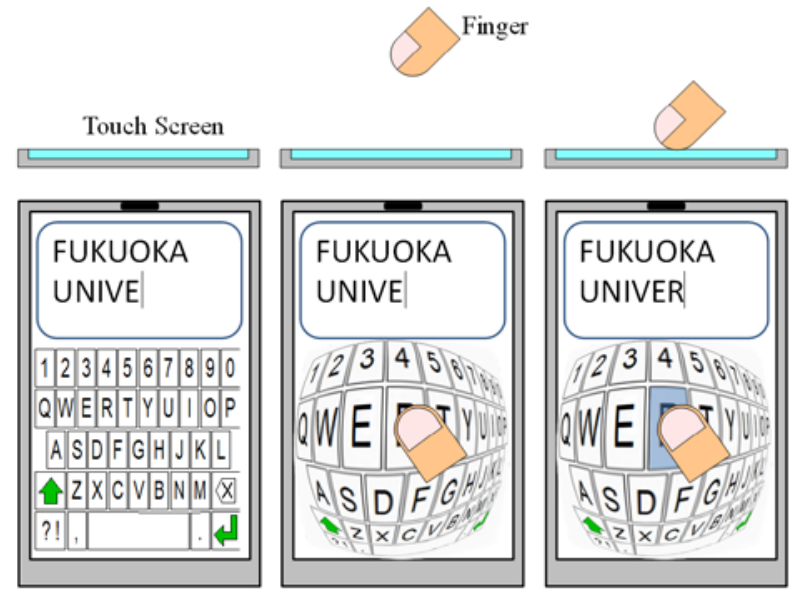

Fig. 1. The goal image of proximity and pressure touch screen in this study. 
easy to push small interface buttons. When the finger touch the sensor, the sensor detects the pressure and its position of the object, and the image is operated by applying a relatively pressure. Therefore, this sensor would assure the functionality and usability of devices using proximity and pressure touch screens.

Previously, we proposed a layered 3D touch screen to detect an object both before and after contact ${ }^{(12)}$. In the sensor, two types of capacitance sensors are layered. The plane-type capacitance electrodes of the top sensor are large in order to increase sensitivity before contact, and the parallel-type capacitance electrodes of the bottom sensor are small in order to realize a high spatial resolution during contact. However, the measurement points of the top sensor are few because the electrodes of the top sensor are large. Thus, the resolution is reduced within the proximity range.

In this paper, we propose a proximity and pressure touch screen method using mutual capacitance measurement. The sensor consists of the separate-type sensor and the capacitance pressure sensor which is parallel-type electrodes. The proposed sensor can combine and separate electrodes on separate-type sensor at proximity range. The coupled electrodes are large so as to increase the sensitivity along the $\mathrm{Z}$-axis, and the separated electrodes increase the resolution of the $\mathrm{X}-\mathrm{Y}$ axes when the object is in close proximity range ${ }^{(13)}$. In addition, the capacitance pressure sensor detects the pressure and center position on contact. We aim to improve the proximity sensitivity along the Z-axis by separate-type sensor, and detect the pressure and precise contact position by pressure sensor. A prototype sensor using this technique detects an object within the proximity range, and detects the pressure and it position.

\section{Principles}

This proposed method is to increase the sensitivity along the Z-axis within the proximity range, and to detect the contact pressure using mutual capacitance measurement. Fig. 2 (a) shows the schematic diagram of the proposed method. The proposed sensor consists of plane-type electrodes $\left(E_{1}\right.$ and $E_{2}$ ) and four parallel-type electrodes which is the pressure sensor. The plane-type electrodes detect the position and multi-point of the object within the proximity range. The parallel-type electrodes detect the pressure and precise center point of the touch. In plane-type capacitance sensors, the capacitance between the electrodes is proportional to their areas. In addition, the shorter the separation between the electrodes, the larger the capacitance measured. However, Z- (a)

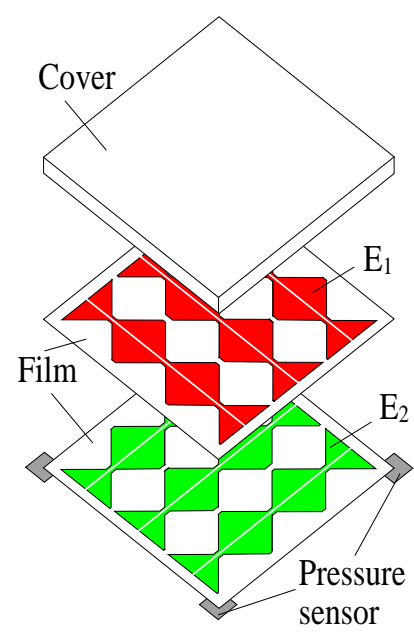

(b)

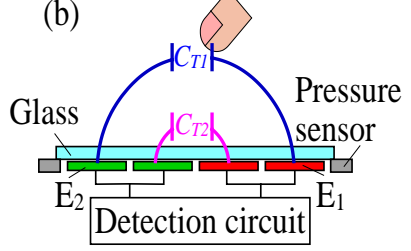

(c)

$\mathrm{E}_{2}$

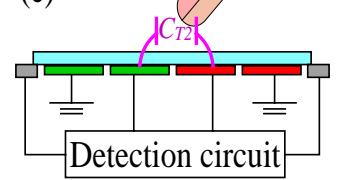

(d)

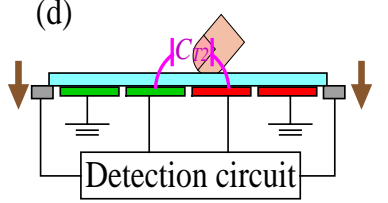

Fig. 2. Principle of the proposed proximity and pressure touch screen. (a) Schematic diagram of proximity and pressure touch screen. (b) Measurement of the coupled electrode. (c) Measurement of the separated electrode. (d) Measurement of the pressure sensor.

axis information is reduced because the electric field passes mainly through the nearby electrodes. On the other hand, the larger the separation between the electrodes, the smaller the capacitance. However, Z-axis information is increased because the electric field passes mainly far away from the electrodes. In the proposed method, the detection sensitivity along the Z-axis is increased by the coupled electrodes. The $\mathrm{X}-\mathrm{Y}$ resolution of the proposed sensor is increased by the separated electrodes when a finger is in close proximity range. Figs. 2(b) and (c) depict each measurement. Fig. 2(b) shows the measurement of the coupled electrodes, and Fig 2(c) shows the measurement of the separated electrodes. In Fig. 2(b), the coupled electrodes measure the capacitance $(C)$. $C_{T}$ is given by

$$
C_{T}=C_{T 1}+C_{T 2}
$$

Here, $C_{T 1}$ is the capacitance between far-spaced electrodes, and $C_{T 2}$ is the capacitance between closely spaced electrodes. When the object is far away, mainly $C_{T 1}$ is changed. Thus, the proposed method can detect an object far away from the sensor because coupled electrodes can detect the change in $C_{T 1}$. In addition, $C_{T 2}$ is changed when the object is near. Thus, it is possible to increase the $\mathrm{X}-\mathrm{Y}$ resolution with separated electrodes because the separation between the electrodes is small. Therefore, we expect that coupled electrodes can increase detection sensitivity along the Z-axis when an object is far away and that separated electrodes can increase $\mathrm{X}-\mathrm{Y}$ resolution when an object is near ${ }^{(13)}$. In addition, four 


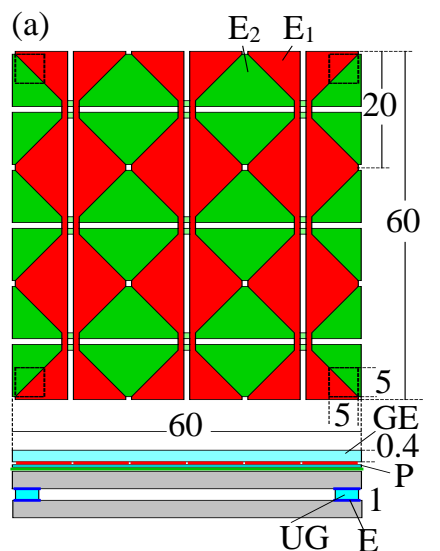

(b)

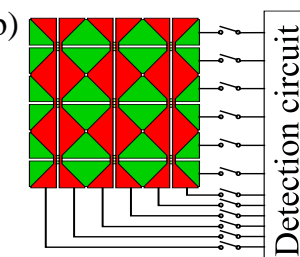

(c)

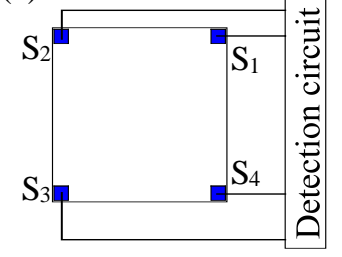

Fig. 3. The proposed sensor (a) Sensor structure. E, $E_{1}$ and $\mathrm{E}_{2}$ : Electrode, GE: Glass epoxy, P: Polyethylene terephthalate, UG: Urethane gel, $\mathrm{S}_{1}-\mathrm{S}_{4}$ : Pressure sensor, Dimensions in $\mathrm{mm}$. (b) Measurement system of the separation-type electrodes. (c) Measurement system of the pressure sensor.

parallel-type electrodes detect the pressure and a precise center point of the touch. Thus, the proposed sensor detects the object using coupled electrodes when an object is far away, and detect the object using separated electrodes when the object is near. The proposed sensor detects the multi point using separated electrodes, and detects the pressure and a precise center point using parallel-type electrodes on contact.

\section{Prototype Sensor}

Fig. 3 shows the structure of the prototype touch screen. A large electrode is required to increase the sensitivity of electrical measurements. However, the separation between the electrodes must be reduced in order to realize high spatial resolution. Thus, we propose a structure wherein the coupled electrodes $\left(E_{1}\right.$ and $\left.E_{2}\right)$ of the plane-type capacitance sensor are large so as to increase the sensitivity before contact. In addition, the electrodes can be separated to realize a high spatial resolution at close proximity range. Thus, the prototype plane sensor consists of $3 \times 3$ coupled electrodes and $6 \times 6$ separated electrodes ${ }^{(13)}$. The width of electrodes in a conventional touch screen is about $5 \mathrm{~mm}$. Therefore, the width of the electrodes on the proposed sensor is larger than in conventional electrodes. A glass epoxy sheet (with a thickness of $0.4 \mathrm{~mm}$, denoted by GE in Fig. 3) is placed on top of the sensor that is isolated from the object. The polyethylene terephthalate (with a thickness of $0.1 \mathrm{~mm}$ denoted by $\mathrm{P}$ in Fig. 3) is placed between $\mathrm{E}_{1}$ and $\mathrm{E}_{2}$ to isolate the electrodes. The electrodes are copper-plated in basic studies. The parallel-type electrodes are set on four corners of the sensor. The size of electrodes of parallel-type

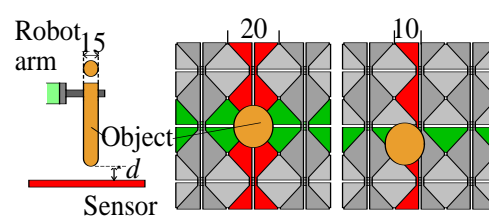

(a) (b) (c)

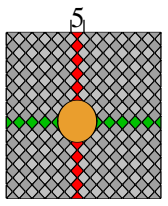

(d)

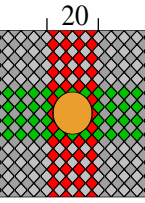

(e)
Fig. 4. Measurement condition.

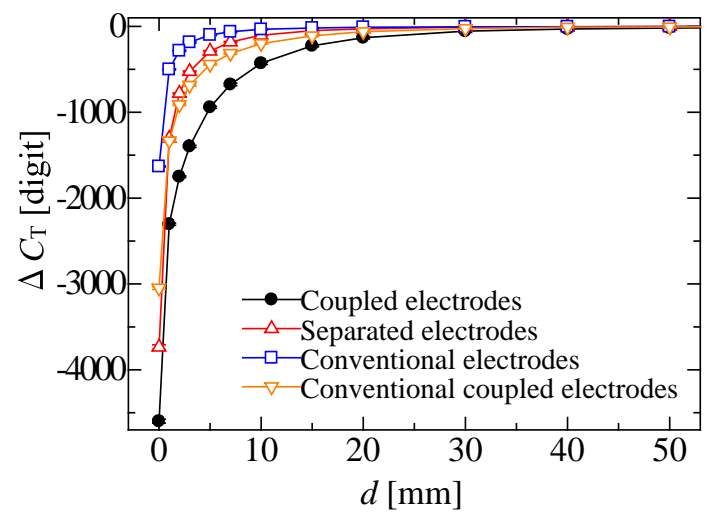

Fig. 5. Relationship between the distance ( $d$ ) and $\Delta C_{T}$.

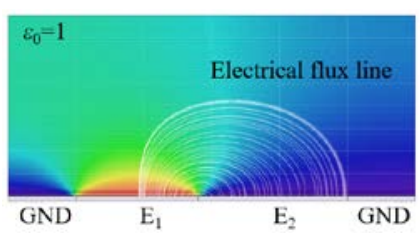

(a)

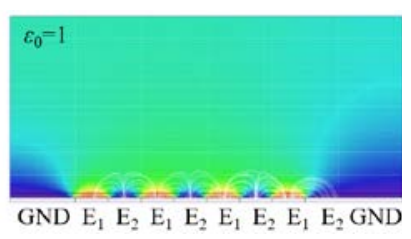

(b)
Fig. 6. Simulated results of electric potential and line of electric force.

electrodes (E) are a square $5 \mathrm{~mm}$ on a side. The urethane gel (with a thickness of $1.0 \mathrm{~mm}$ denoted by UG in Fig. 3) is set between parallel-type electrodes. The capacitances are measured by a control circuit which consists of an analog switch, microcomputer, and capacitance measurement IC (Analog Devices, AD7142). The AD7142 is a capacitanceto-digital converter (16-bit). The measured data (16-bit) are captured by the PC. The unit of the measurement values indicates A/D converted digital values (digit). The electrodes not used for measurement are connected to a shield, which is either the ground or has an equal potential to that of the measurement signal. The object is a grounded conductor $(\varnothing 15 \mathrm{~mm}$ ) which is the finger model in this study. The object was attached to a robot arm.

\section{Results and Discussion}

\subsection{Proximity Measurement}

We show the results of proximity measurement with the 


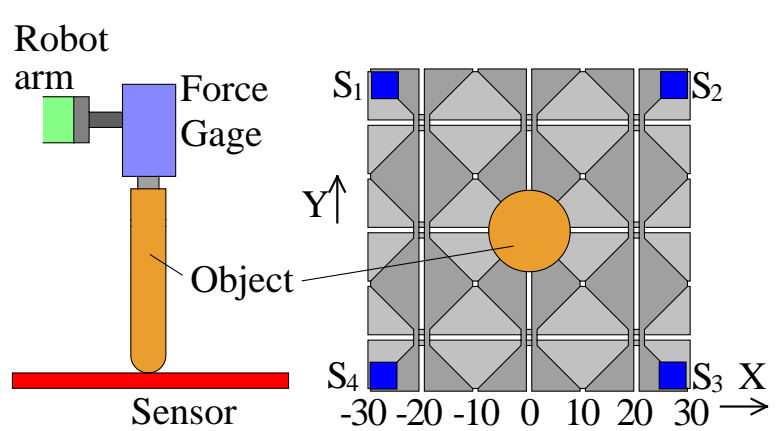

Fig. 7. Measurement condition.

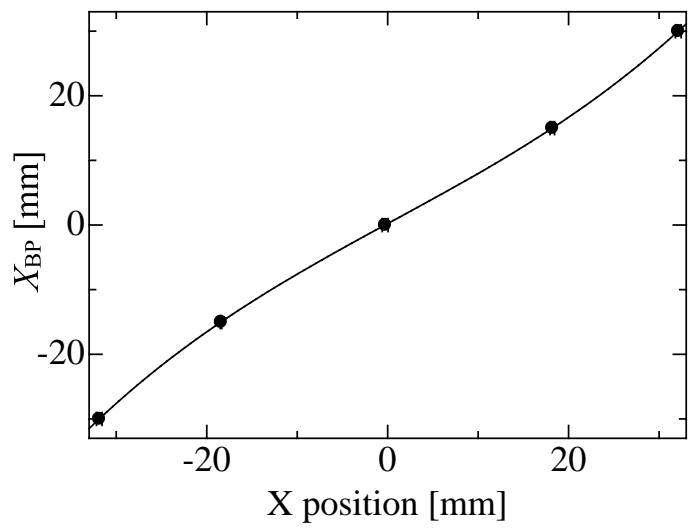

Fig. 8. Relationship between the $\mathrm{X}$ position and $X_{B P}$.

proposed electrodes and conventional electrodes to detect an object along the Z-axis ${ }^{(13)}$. The distance $(d$, positioned from 0 to $50 \mathrm{~mm}$ ) between the sensor and the object was changed by the robot arm (Fig. 4(a)). Fig. 4(b) shows the position of the object and the proposed sensor where the coupled electrode width (EW) is $20 \mathrm{~mm}$, and Fig. 4(c) shows the position of the object and the proposed sensor where the separated electrode (EW : $10 \mathrm{~mm}$ ), and Fig. 4(d) shows the position of the object and the conventional sensor (EW : 5 $\mathrm{mm}$ ), and Fig. 4(e) shows the position of the object and the conventional coupled sensor (EW : $20 \mathrm{~mm}$ ). Here, the colored electrodes are measurement electrodes. Fig. 5 shows the variations $\left(\Delta C_{T}\right)$ measured by each electrode. In Fig. 5, $\Delta C_{T}$ changes according to $d$. Thus, the sensor can detect an object before contact using $\Delta C_{T} . \Delta C_{T}$ in the coupled proposed sensor is changed from far away. $\Delta C_{T}$ in a conventional sensor is changed from nearby. Fig. 6 shows the 2D map of electric potential and line of electric force of proposed coupled sensor and conventional coupled sensor by simulation. The potential value of $E_{1}$ is $1 \mathrm{~V}$, and the potential value of $E_{2}$ is $0 \mathrm{~V}$. The line of electric force of proposed coupled sensor is through far away. This is the reason for the increased detection sensitivity along the Z-axis when increasing the size of the electrodes. In this experiment, the coupled proposed electrodes can detect an object within

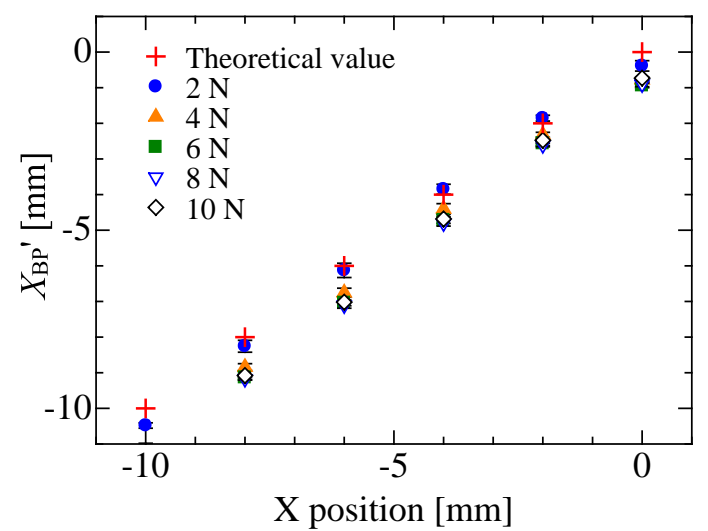

(a)

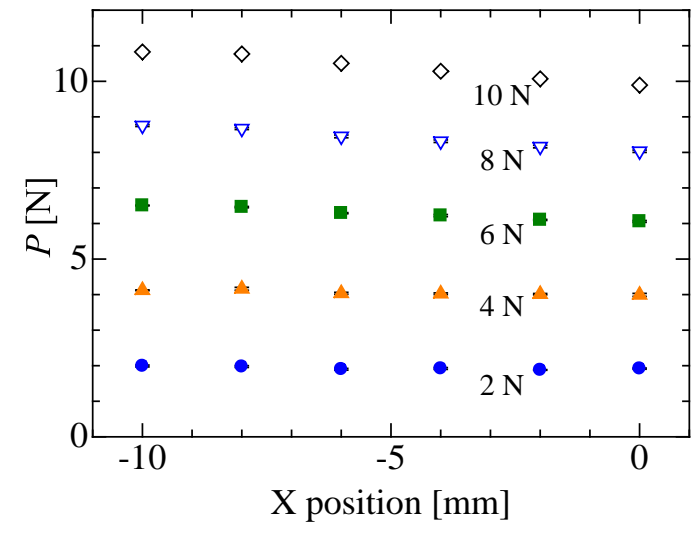

(b)

Fig. 9. Measurement results. (a) Relationship between $X_{B P}$ and $Y_{B P}$ '. (b) Relationship between $\mathrm{X}$ position and $P$.

approximately $40 \mathrm{~mm}$. Separated electrodes can detect an object within approximately $30 \mathrm{~mm}$. Conventional electrodes can detect an object within approximately $10 \mathrm{~mm}$. Conventional coupled electrodes can detect an object within approximately $30 \mathrm{~mm}^{(13)}$.

\subsection{Contact Measurement}

We evaluated the contact measurement using the parallel-type electrodes to detect a precise center point on contact. Fig. 7 shows the position of the object and the proposed sensor. The object was moved onto an arrow \pm 30 $\mathrm{mm}$ from the center of the sensor in the $\mathrm{X}$ direction. The contact condition was detected by a force gauge. The pressure is $2 \mathrm{~N}$. The pressure between the sensor and the object was changed by the robot arm. We calculated the $\mathrm{X}-\mathrm{Y}$ position of an object on the contact using each $\Delta C_{B}$ for each electrode. The $\mathrm{X}$ position $\left(X_{B P}\right)$ and $\mathrm{Y}$ position $\left(Y_{B P}\right)$ were calculated by the following equation using $\Delta C_{B}$ :

$$
\begin{gathered}
X_{B P}=\frac{\Delta C_{B 1}-\Delta C_{B 2}-\Delta C_{B 3}+\Delta C_{B 4}}{\Delta C_{B 1}+\Delta C_{B 2}+\Delta C_{B 3}+\Delta C_{B 4}} \\
Y_{B P}=\frac{\Delta C_{B 1}+\Delta C_{B 2}-\Delta C_{B 3}-\Delta C_{B 4}}{\Delta C_{B 1}+\Delta C_{B 2}+\Delta C_{B 3}+\Delta C_{B 4}}
\end{gathered}
$$


Here, $\Delta C_{B 1}-\Delta C_{B 4}$ is the capacitance variations of each pressure sensor $\left(\mathrm{S}_{1}-\mathrm{S}_{4}\right)$. Fig. 8 shows $X_{B P}$ which is calculated using Eq. (2). The horizontal axis was the object position (X position) using the robot arm. In Fig. $8, X_{B P}$ is changed according to the $\mathrm{X}$ position of the object. However, the error of $X_{B P}$ are caused. Thus, we corrected $X_{B P}$ by the least squares method using Eq. (4).

$$
X_{B P}{ }^{\prime}=k_{0}+k_{1} X_{B P}+k_{2} X_{B P}{ }^{2}+k_{3} X_{B P}{ }^{3}
$$

Here, $k_{i}(i=0-3)$ indicates the coefficients. We evaluated the corrected $X_{\mathrm{BP}}\left(X_{B P}{ }^{\prime}\right)$. The object was moved onto $\pm 10 \mathrm{~mm}$ from the center of the sensor in $2 \mathrm{~mm}$ increments in the $X$ direction. The pressure are from $2 \mathrm{~N}$ to $10 \mathrm{~N}$. Fig. 9(a) shows the relationship between the calculated $X_{B P}$ ' and $\mathrm{X}$ position. Thus, it is possible to detect the center position of object on $X_{B P}$ ' using $\Delta C_{B}$. In this experiment, the average error (AE) of $X_{B P}$ ' when the pressure is $2 \mathrm{~N}$ is $0.26 \mathrm{~mm}, \mathrm{AE}$ of $X_{B P}$ ' of $6 \mathrm{~N}$ is $0.94 \mathrm{~mm}, \mathrm{AE}$ of $X_{B P}$ ' of $10 \mathrm{~N}$ is $0.87 \mathrm{~mm}$. Here, $\mathrm{AE}$ of $2 \mathrm{~N}$ is low because Eq. (4) used the value of $2 \mathrm{~N}$. However, we think that the error can be reduced by correcting a value of 2 $\mathrm{N}$ because the pressure change to strong from weak pressure.

Next, we evaluated the contact measurement using the parallel-type electrodes to detect the pressure on contact. The pressure $(P)$ was calculated by the following least squares method using the values of each sensor $\left(\mathrm{S}_{1}-\mathrm{S}_{4}\right)$ :

$$
P=f\left(\Delta C_{A}\right)+f(D)
$$

Here, $\Delta C_{A}$ is sum of $\Delta C_{B 1}-\Delta C_{B 4}$, and $D$ is the distance from the center of the electrodes. Fig. 9(b) shows the calculated pressure using Eq. (5) and $X$ position. In Fig. 9(b), $P$ is changed according to the pressure. In this experiment, $\mathrm{AE}$ of $2 \mathrm{~N}$ is $0.068 \mathrm{~N}, \mathrm{AE}$ of $6 \mathrm{~N}$ is $0.27 \mathrm{~N}, \mathrm{AE}$ of $10 \mathrm{~N}$ of $0.11 \mathrm{~N}$. Thus, the proposed sensor can detect the pressure on each position.

We compared contact measurement of $\mathrm{X}$-axis with the plane separation-type electrodes and the parallel-type electrodes. The values of coupled electrodes and separated electrodes of the plane separation-type electrodes were calculated on contact point $(0 \mathrm{~N})$, and the values of pressure sensor were calculated on 2N. Fig. 10 shows each result. $X_{P}$ is changed according to the $\mathrm{X}$ position (Theoretical values). In this experiment, $\mathrm{AE}$ of coupled electrodes is $1.1 \mathrm{~mm}$ and the standard deviation (SD) is $0.079 \mathrm{~mm}, \mathrm{AE}$ of separated electrodes is $0.49 \mathrm{~mm}$ and $\mathrm{SD}$ is $0.017 \mathrm{~mm}$, and $\mathrm{AE}$ of the pressure sensor is $0.26 \mathrm{~mm}$ and $\mathrm{SD}$ is $0.13 \mathrm{~mm}$. In this experiment, $\mathrm{AE}$ of the pressure sensor is better than $\mathrm{AE}$ of coupled and separated electrodes. However, SD of coupled and separated electrodes are better than SD of the pressure sensor.

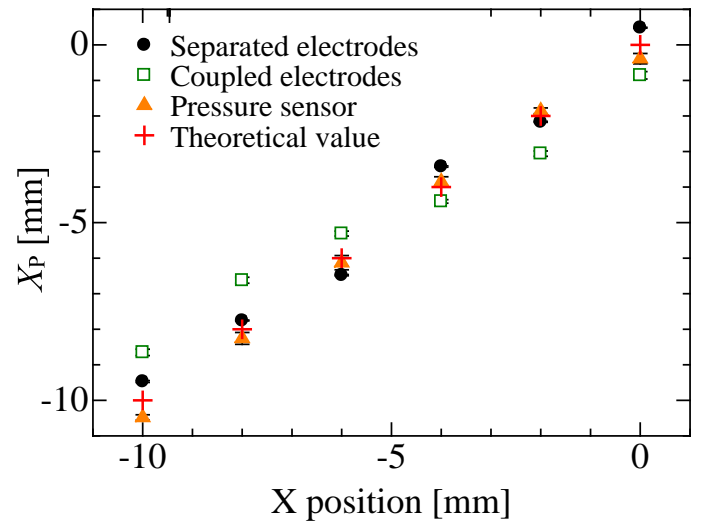

Fig. 10. Relationship between the $\mathrm{X}$ position and $X_{P}$.

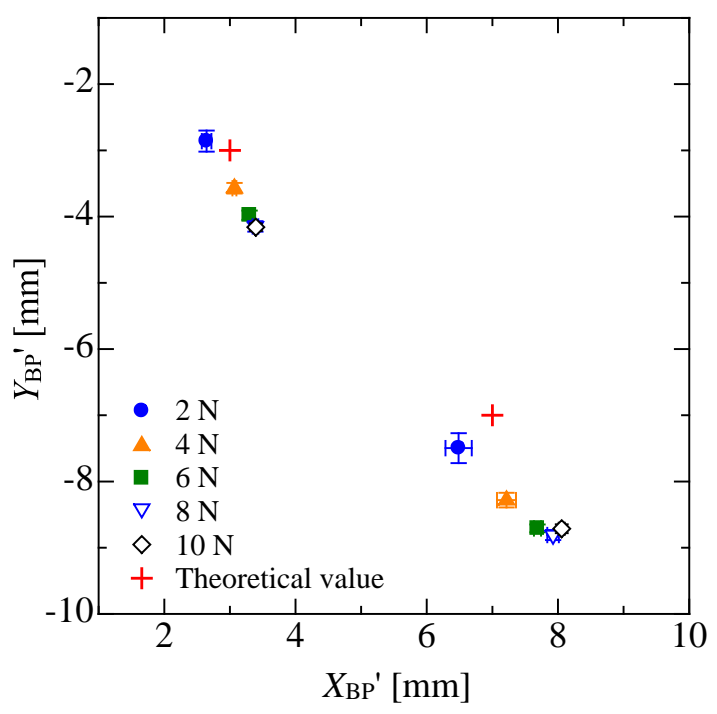

(a)

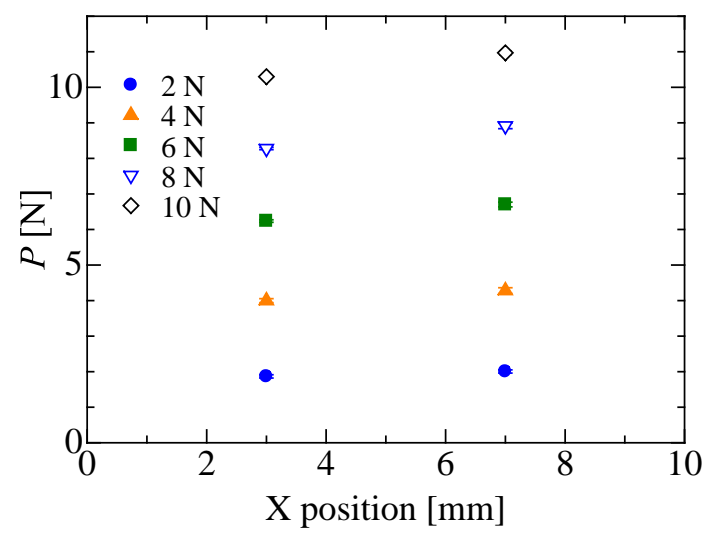

(b)

Fig.11. Measurement results. (a) Relationship between $X_{B P}$ ' and $Y_{B P}$ '. (b) Relationship between $\mathrm{X}$ position and $P$.

Next, we evaluated the X-Y position and the pressure measurement using pressure sensor. The object was set the point at X-Y coordinates $(3,-3)$ and $(7,-7)$ on the sensor. Fig. 10(a) shows the calculated X-Y coordinate position using Eq. (2), (3) and (4). Fig. 10(b) shows the calculated pressure 
using Eq. (5). The X-Y position and the pressure can be detected using this proposed sensor. In this experiment, $\mathrm{AE}$ of $\mathrm{X}-\mathrm{Y}$ position of $2 \mathrm{~N}$ is $0.54 \mathrm{~mm}, \mathrm{AE}$ of $\mathrm{X}-\mathrm{Y}$ position of $6 \mathrm{~N}$ is $1.4 \mathrm{~mm}$, and $\mathrm{AE}$ of $\mathrm{X}-\mathrm{Y}$ position of $10 \mathrm{~N}$ is $1.6 \mathrm{~mm}$. $\mathrm{AE}$ of pressure is $0.38 \mathrm{~N}$. Therefore, the coupled electrodes detect the object position when the object when the object is far away. The separated electrodes detect the object position when the object is near and on contact. In addition, the pressure sensor detects the pressure and its center position on contact. Here, the separated electrodes detect multi point on contact because the pressure sensor cannot detect multi point. We think that it is possible to measure from proximity range to contact by complementing each other using the separation-type capacitive sensor and pressure sensor in this way.

\section{Conclusions}

We propose a proximity and pressure touch screen method using mutual capacitance measurement. The sensor consists of the separate-type sensor and pressure sensor. The proposed sensor can combine and separate electrodes on separate-type sensor at proximity range. The coupled electrodes are large so as to increase the sensitivity along the $\mathrm{Z}$-axis, and the separated electrodes increase the resolution of the $\mathrm{X}-\mathrm{Y}$ axes when the object is in close proximity range. In addition, the pressure sensor detects the pressure and center position on contact. A prototype sensor using this technique detects an object within the proximity range, and detects the pressure and it position. Thus, we think that the proposed sensor will be one of useful methods as a touch screen.

\section{Acknowledgment}

This work was supported in part by JSPS KAKENHI Grant Number JP 15K16043.

\section{References}

(1) D. K. Kim, J. H. Kim, M. J. Kwon, and Y. H. Kwon : “A touchpad for force and location sensing”, ETRI J. vol. 32, No. 5, pp. 722-728. 2010

(2) P. Dietz and D. Leigh : "DiamondTouch: A multi-user touch technology”, Proceedings of UIST '01, pp. 219226, 2001

(3) H. K. Kim, S. Lee, and K. S. Yun : "Capacitive tactile sensor array for touch screen application”, Sensors and
Actuators A, vol. 165, No. 1, pp. 2-7, 2011

(4) K. Kyoung, K. Yuge, and R. Hattori : "Single-layered surface / projected hybrid capacitive touch panel”, Proceedings of IMID 2011, pp. 174-175, 2011

(5) G. Barrett and R. Omote : "Projected-capacitive touch technology”, Inf. Disp., Vol. 26, No. 3, pp. 16-21, Mar. 2010.

(6) O. Hilliges, S. Izadi, A. D. Wilson, S. Hodges, A. G. Mendoza, and A. Butz : "Interactions in the air: adding further depth to interactive tabletops”, Proceedings of UIST '09, pp. 139-148, 2009

(7) S. Kratz and M. Rohs : "HoverFlow: Expanding the design space of around-device interaction”, Proceedings of MobileHCI '09, 2009)

(8) http://www.cypress.com/?rID=60561

(9) S. Heo and G. Lee : "Force gestures: augmented touch screen gestures using normal and tangential force”, Proceedings of CHI EA '11, pp.1909-1914, 2011

(10)E. Furuki and Y. Kikuchi : "Approach to Commercialization of RakuRaku Smartphone”, FUJITSU, Vol. 63, No. 5, pp. 548-554, 2012

(11) http://www.apple.com/jp/shop/buy-iphone/iphone6s

(12) S. Tsuji, T. Kohama : “A Layered 3D Touch Screen Using Capacitance Measurement”, IEEE Sensors Journal, Vol. 14, No. 9, pp. 3040-3045, 2014

(13) S. Tsuji, T. Kohama : “A proximity touch screen method using separation-type electrodes”, Sensors and Actuators A, Vol. 252, pp. 1-6, 2016 